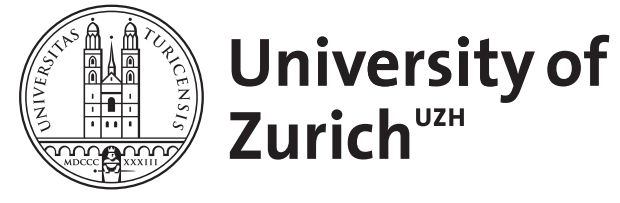

\title{
About the pathophysiology of acute unilateral vestibular deficit - vestibular neuritis (VN) or peripheral vestibulopathy (PVP)?
}

Uffer, Denis S ; Hegemann, Stefan C A

DOI: https://doi.org/10.3233/VES-160581

Posted at the Zurich Open Repository and Archive, University of Zurich ZORA URL: https://doi.org/10.5167/uzh-126077

Journal Article

Accepted Version

Originally published at:

Uffer, Denis S; Hegemann, Stefan C A (2016). About the pathophysiology of acute unilateral vestibular deficit - vestibular neuritis (VN) or peripheral vestibulopathy (PVP)? Journal of Vestibular Research, 26(3):311-317.

DOI: https://doi.org/10.3233/VES-160581 
About the pathophysiology of acute unilateral vestibular deficit

- vestibular neuritis (VN) or peripheral vestibulopathy (PVP)?

Uffer, Denis; Hegemann Stefan C. A.

University of Zurich, Faculty of Medicine; 


\section{Abstract}

Objective: To determine whether patients with acute unilateral peripheral vestibulopathy (PVP), often called "vestibular neuritis/neuronitis or neuropathy" (VN) have a vestibular lesion pattern consistent with the distribution of the neurological afferents.

Background: Much is known about the clinical nature of PVP, however less so about its etiology and pathogenesis. Due to the frequency in which VN is used to describe the syndrome, an inflammation of the vestibular nerve or of one of its branches is often assumed to be the cause of PVP, though there is insufficient data so far to support this assumption.

Methods: We conducted a retrospective study of 25 patients who had presented to our clinic with PVP and had tested all vestibular receptor organs shortly after start of symptoms. We analysed their vestibular lesion patterns in order to determine whether they were consistent with the neuritis hypothesis $(\mathrm{NH})$.

Results: The lesion patterns varied conspicuously, $76 \%$ did not abide by the innervation pattern to the $\mathrm{NH}$ and only $24 \%$ did having either definite $(16 \%)$ or probable $(8 \%) \mathrm{VN}$.

Conclusion: These results should remind us to be careful before jumping to quick conclusions about the pathogenetic nature of PVP. With any reason to question VN as the only cause of PVP, we should reconsider the treatment approach to PVP. If the cause probably or even possibly lies inside the vestibular labyrinth, an intratympanic steroid injection might prove to be a more effective measure, even in firstline treatment. If the etiology is unsure, a combination of systemic and intratympanic steroid treatment may be adequate. 


\section{Introduction}

The symptoms of an acute vestibular syndrome are well described in the literature. They are mainly due to a unilateral peripheral vestibulopathy (PVP), if central lesions are excluded are most commonly named vestibular neuritis, neuronitis or neuropathy- for a short historical review see Reker [34]. Since there are no formal diagnostic criteria there is some variation in the definition, which poses a challenge when trying to compare studies from different authors or clinics[3, 37]. PVP manifests itself in the acute onset of spinning vertigo lasting many hours or several days, often accompanied by nausea and vomiting $[14,20]$. The patients usually present with gait instability[14] and a falling tendency toward the affected side. The clinician normally detects a horizontal nystagmus beating toward the healthy side obeying Alexander's law. Caloric and head impulse testing show hypofunction of the horizontal semicircular canal (hSCC)[37]. The frequently occurring ocular tilt reaction[22] toward the affected side which can be observed directly through fundoscopy or be inferred from testing the subjective visual vertical (SVV)[24] is indicative of an utricular hypofunction as is an asymmetry ratio (AR) in ocular vestibular evoked myogenic potentials (oVEMPs). The saccular afferents from the vestibular organ as tested through cervical VEMPs frequently remain unaffected[14], but can also be dysfunctional[30, 45]. The frequent sparing of the sacculus previously led to the conclusion that vestibular neuritis chiefly affects the superior vestibular nerve whilst sparing its inferior half in most cases[14]. Another explanation for these findings calls attention to the fact that patients who only have pathologies in their posterior SCC (pSCC) and/or sacculus are less likely to be diagnosed as having PVP due to the absence of a spontaneous nystagmus (SPN) or pathological caloric test results[21, 26].

At the time the etiological hypothesis of superior / inferior / total vestibular neuritis (henceforth: neuritis hypothesis, NH) was proposed it was reasonable to assume that the dysfunction in PVP follows a neuroanatomical pattern, since most routine testing included only one receptor per nerve branch (caloric for hSCC i.e. superior vestibular nerve, cVEMP for sacculus i.e. inferior vestibular nerve). However, such assumptions need to be re-evaluated in the advent of new diagnostic methods that might offer new insights into the pathogenesis of PVP. For about a decade, it has now been possible to assess both utricular and saccular function in clinical practice using oVEMP (introduced in 2007[35]) and cVEMP (introduced in 1992[35]). The video head impulse 
test (vHIT), introduced for hSCC testing in 2009[5, 28] and for vertical SCC testing in 2013[27], which serves as an alternative to the more expensive, time-consuming and more discomforting magnetic search-coil HIT, first described for testing all SCC in 1998[10] allows for a quick assessment of all SCC receptors separately. Given the availability of these new precise methods it is sensible to test the NH against all receptors, that is, if the NH is true, PVP should either show a hypofunction of all receptors of the superior vestibular nerve, all receptors of the inferior vestibular nerve or both. In other words, the lesion pattern of the vestibular receptors in PVP should be the same as the innervation pattern of the vestibular organ.

To our knowledge an analysis of the lesion patterns in PVP with the aim of testing the NH has not been performed so far. The goal of our study was to map the lesion patterns of our PVP patients in order to test whether they are the same as the innervation patterns.

In a retrospective study was according to the declaration of Helsinki and approved by the local ethics committee, we analysed the lesion patterns of the patients who had presented and been treated for PVP in our clinic over the last years.

\section{Etiology and pathogenesis of PVP: The evidence so far}

The research on the etiology and pathogenesis of PVP is variegated. There are many contradictory findings, some of which support the NH while others do not or even contradict it. The many terms - vestibular neuritis / neuronitis / neuropathy / neuronopathy, acute vestibulopathy, acute vestibular neurolabyrinthitis, acute labyrinthitis ${ }^{1}$ - often used interchangeably to describe PVP, testify to the fact that there is still much to be discovered about the nature of the disease.A less disputed issue is the fact that PVP is often preceded by a viral infection of the upper respiratory tract[37] or the higher prevalence (compared to controls) of Herpes viruses in the eighth and other cranial nerves and their neurons[1, 12, 17, 41] as well as in the saliva of patients with PVP[33]. One animal study also supports the etiopathogenetic role of Herpes viruses, but points to histopathological changes in both the vestibular nerve and labyrinth[13]. HSV-1 has also be detected in the labyrinth [2]

\footnotetext{
${ }^{1}$ This term is misleading, because it is usually used to describe a combined vestibulo-cochlear deficit. (Baloh, Clinical Practice 2003)
} 
The anatomical evidence of a vestibulo-facial nerve anastomosis is one way of explaining the presence of those viruses in the vestibular neurons.[32] However, the prevalence as described in those studies is insufficient to support Herpes viruses as the only etiological factor. More likely those findings point to the fact that PVP may have a multifactorial etiology. Alternatively they raise the question whether PVP might be, rather than a single pathological entity, a catch-all basket for a group of diseases with similar symptoms that do not match another known diagnostic entity.

Often the case for the NH is made by presenting PVP as analogous to Bell's palsy, which is also thought to be caused by a reactivation of viruses that subsequently leads to a nerve oedema and a loss of function[2, 15]. However reasonable this analogy may seem, there are two important differences: Firstly, corticosteroids have been found to be reasonably effective in the treatment of Bell's palsy[15], while their effect in treating PVP is less established [16, 43]. This might be suggestive of a distinctive pathogenetic mechanism rather than a similarity. Secondly, while the Bell's palsy analogy is a justified model to consider, other equally plausible analogies should also be taken into account. This includes, for instance, Herpes labialis, in which the reactivation of viruses - often proposed as a possible pathomechanism in PVP - leads to pathological changes in the sensory end organ (skin). This analogy would support a pathogenetic model with intralabyrinthic changes, rather than an isolated neuritis.

Other evidence cited in favour of the NH includes MRI studies and histopathological analyses. There is one MRI double case report that mentions changes on the vestibular nerve (but not in the labyrinth) in two patients, 7 and 11 days after symptom onset, respectively[25]. Besides being a case report, findings made many days after the first symptoms need to be considered with much care. Moreover, this study contradicts two othersthat looked at MRI's of 8 and 60 patients with PVP, respectively and found no signs of a neuritis[23, 40]. Histopathological studies have found that there was evidence of viral infections and subsequent loss of neurons in the vestibular nerve. However, they also found significant changes in the labyrinth: There was a kind of "epithelialization" on the otolith maculae as well as in the cristae of the SCC[4]. An animal study confirms the evidence of a viral infection on both the vestibular nerve and labyrinth[13]. 
The fact that benign paroxysmal positional vertigo (BPPV) is more prevalent in patients with a history of PVP also supports a pathogenetic model with the involvement of an inthralabyrinthine lesion (some $20 \%$ of patients with a history of PVP will develop BPPV in their lifetime[20]).

As a whole, these findings do not strongly support the NH. It would therefore at least be prudent to consider the involvement of the vestibular labyrinth in the pathogenesis of PVP.

The main goal of this study is to determine how many of the patients with PVP present with an intralabyrintine lesion pattern. In other words: In what part of patients is the vestibular lesion pattern at odds with the vestibular innervation anatomy? 


\section{Materials and Methods}

The patient database of our clinic was searched for patients aged 18-80 who had presented between January 2006 and April 2014 and for whom a diagnosis of PVP was considered due to their clinical symptoms $(n=202)$. After applying the exclusion criteria, 25 patients qualified for our study.

Exclusion criteria were as follows (see also figure 1): patients with a different final diagnosis ( $\mathrm{n}=60,7$ Menière or migraine; 42 with additional hearing loss, thus labyrinthitis; 11 with vestibular schwannoma), patients on whom relevant diagnostic tests were not performed within 10 days of symptom onset and within a maximum of 3 days after the beginning of treatment with corticosteroids $(n=117)$. There were no patients in this sample with vertigo of central cause, who we would also have excluded from our study.

We included only patients who had tests performed on all their SCC receptors and the utricle. There were 8 patients without cVEMP's, thus without saccular diagnostics.

Our approach to classifying patients as having PVP is one of a diagnosis of exclusion[39]. This means we included all patients with prolonged acute vertigo and nystagmus without hearing loss, for whom no other diagnosis or more likely cause was found.

In order to understand whether receptors with equal innervations were similarly affected - which would support the $\mathrm{NH}$ - we introduced a scale of 4 different dysfunction levels (DL) for each receptor (see table 1). Those levels are to be understood as follows: $0=$ no dysfunction, $1=$ slight dysfunction or possible false positive, $2=$ definite dysfunction, $3=$ strong dysfunction or total loss of function.

The dysfunction levels for the vertical SCC's were calculated on the basis of their respective HIT gains. If for a specific receptor there were clear corrective saccades despite the presence of a normal gain (3 cases, i.e. covert saccades), they were classified as strongly dysfunctional. The DL of the hSCC was calculated from either the HIT alone or the average of HIT, DVA and caloric CP, in case those latter tests had been performed. The DL's for the utricle were similarly calculated by using either one or an average of the test results from the SVV[42], the ocular torsion (OT)[9, 11] and oVEMP. Finally, the saccular DL was calculated on the basis of cVEMP's[36, 44]. 
We then classified the patients according to whether their lesion pattern supported the NH (i.e. the lesion pattern was the same as the innervation pattern), using a second set of groups (see also fig. 2 and 3 for examples): definite neuritis-like pattern (NP, see also figure 2), likely NP, likely ILP (inthralabyrinthine lesion pattern), definite ILP (table 2, see also figure 3). Strictly speaking, the occurrence of an ILP in a patient would contradict the NH. An inthralabyrinthine lesion though, could theoretically present as a NP, which is why the occasional occurrence of a NP does not, by itself, condradict the inthralabyrinthine lesion thesis. Therefore, if the NH is true, we would expect an overwhelming majority of the patients to present with a NP, allowing for the few remaining cases to be explained as measurement errors. 


\section{Results}

Among our 25 patients $19(76 \%)$ had a lesion pattern that looked more like an ILP than a NP (see figure 4). 9 of those had a definite ILP, while another 10 likely do not fit the NH. Only 6 patients (24\%) had a NP, potentially supporting the NH, 4 (16\%) of which had a definite NP, the remaining 2 a likely NP. Roughly half of the patients with a definite ILP had major dysfunction differences in the receptors of the superior vestibular nerve, the other half had dysfunction differences in the receptors of the inferior vestibular nerve (see table 3 ).

\section{Inter-method-validity of diagnostic methods for the same vestibular receptor}

In order to validly use different measurement methods for the same receptor, it is important that those measurements deliver similar results. Below are the numbers of inter-measurementdiscrepancies for the different methods to assess the hSCC and utricular function. Two measurements for the same receptor were seen as significantly discrepant when the resulting DL scores differed by more than 1 DL point.

$\underline{h S C C}$

In 2 out of 23 cases where more than the HIT was performed those tests significantly differed from one another (by more than 1 DL point).

\section{$\underline{\text { Utricle }}$}

In 3 out of 15 cases where more than one test for the utricle was performed those results significantly differed from one another (twice by 2 DL points, once by 3 DL points).

All in all, when more than one method was used to assess a receptor function in our study population, it can be said that these methods lead to fairly similar results, meaning that they were sufficiently conclusive to draw further conclusions from them. 


\section{Discussion}

The results of our study do not support the $\mathrm{NH}$ since about 3/4 our patients had an intralabyrinthine lesion pattern inconsistent with an isolated nerve lesion.

Our study does not prove that the only location there can be a lesion causing PVP is the vestibular labyrinth, neither does it reject the $\mathrm{NH}$ altogether. However, our results do suggest that there could be other loci affected by PVP in addition to the vestibular nerve.

Another model to consider is that PVP consists of a combined vestibular neuro-labyrinthitis sparing the cochlea. This theory would account for the tendency among certain patients to present with a NP while the ILP found in the others can be explained by an intralabyrinthine inflammation that does not follow the innervation pattern.

Consequently, it raises the question whether systemic corticosteroids are the best approach to treat PVP. It is well documented, that even high-dosage i.v. corticosteroids only barely reach the perilymph at very low concentrations, while intratympanic delivery would be much more effective[6-8, 31], not to mention the reduction of systemic adverse effects. And meta-analyses as well as recent studies do not yet show a clear evidence for efficacy of systemic steroid treatment $[16,18,19]$. In sudden sensorineural hearing loss, where a neuritis is rarely assumed to be the cause, intratympanic corticosteroid application is widely accepted for salvage treatment with sufficient evidence in several studies including meta-analyses[38].

Furthermore, an intralabyrinthic lesion theory would give a more appealing explanation as to why patients with a history of PVP are more likely to suffer from BPPV: It is conceivable that an inflammation of the utricle may cause otoliths to dissociate from the receptor, while the $\mathrm{NH}$ fails to address this issue.

A recent study by Magliulo et al.[29] looked at 1-year follow-up results of UVD patients in their oVEMP, cVEMP and vHIT measurements showing acute-phase findings similar to ours: Half of their patients had incomplete involvement of vestibular receptors of their superior and/or inferior vestibular nerve. However, the authors remained true to the $\mathrm{NH}$ and argue for the existence of even more subgroups of vestibular neuritis than the two (three) described so far, superior and inferior vestibular neuritis (or the combination thereof). However, applying the principle of par- 
simony it might be more accurate to consider an intralabyrinthic lesion as a possible cause of PVP.

\section{Limitations}

There are several limitations to our study, the main ones being the small sample size and the retrospective study design.

Other criticism may concern to the introduction of our classification system of vestibular receptor dysfunction with four different DL's. We think though that the use of such semi-quantifiable values is justifiable: The idea of introducing DL's was developed to apply enough spacing between a normal vestibular receptor function and a clear dysfunction in order to judge whether the patient's lesion pattern is in accordance with the $\mathrm{NH}$ or not.

Another limitation is the use of different receptor function measurements in dependence of their availability for each patient. Since our investigation was of qualitative rather than quantitative nature, we think that this does not inflict on the conclusions of our study. However, this certainly is an issue, which we would address in a prospective study design.

Another criticism that might emerge is that neuritis does not need to affect all receptors similarly. In light of other known neuritic lesions of cranial nerves that lead to a more or less homogenous dysfunction of the affected sensory or motor organ (i.e. Bell's Palsy, optic neuritis, oculomotor neuritis), such criticism does not strike us as justified.

\section{Conclusions}

To our knowledge, this is the first study to address the issue of lesion patterns in patients with PVP by using tests of all 5 vestibular receptors aiming at proving or disproving the neuritis hypothesis. Our findings, although limited due to the study design and small sample size, indicate that it might be premature to assume that all PVP is caused by neuritis. Further research into lesion patterns in PVP is needed to clarify this issue.

If our suspicion - that a significant part of PVP patients (if not all of them) has an intralabyrinthine lesion or inflammation - is true, it may have important implications on the treatment approach to PVP. Since even high dose i.v. corticosteroids are known to insufficiently penetrate the 
blood-labyrinth barrier, administration of intratympanic steroids could be a more effective choice to treat affected vestibular endorgan lesions. In case of individually not clearly defined lesions a combination of systemic and intratympanic treatment could be an alternative approach with maybe lower doses of systemic steroids. 


\section{References}

[1] V. Arbusow, M. Strupp, R. Wasicky, A.K. Horn, P. Schulz and T. Brandt, Detection of herpes simplex virus type 1 in human vestibular nuclei [In Process Citation], Neurology 55 (2000), 880-882.

[2] V. Arbusow, D. Theil, M. Strupp, A. Mascolo and T. Brandt, HSV-1 not only in human vestibular ganglia but also in the vestibular labyrinth, Audiol Neurootol 6 (2001), 259-262.

[3] R.W. Baloh, Clinical practice. Vestibular neuritis, N Engl J Med 348 (2003), 1027-1032.

[4] R.W. Baloh, A. Ishyama, P.A. Wackym and V. Honrubia, Vestibular neuritis: clinicalpathologic correlation, Otolaryngol Head Neck Surg 114 (1996), 586-592.

[5] K. Bartl, N. Lehnen, S. Kohlbecher and E. Schneider, Head impulse testing using videooculography, Ann N Y Acad Sci 1164 (2009), 331-333.

[6] P.A. Bird, E.J. Begg, M. Zhang, A.T. Keast, D.P. Murray and T.J. Balkany, Intratympanic versus intravenous delivery of methylprednisolone to cochlear perilymph, Otol Neurotol 28 (2007), 1124-1130.

[7] P.A. Bird, D.P. Murray, M. Zhang and E.J. Begg, Intratympanic versus intravenous delivery of dexamethasone and dexamethasone sodium phosphate to cochlear perilymph, Otol Neurotol 32 (2011), 933-936.

[8] S.S. Chandrasekhar, R.Y. Rubinstein, J.A. Kwartler, M. Gatz, P.E. Connelly, E. Huang and S. Baredes, Dexamethasone pharmacokinetics in the inner ear: comparison of route of administration and use of facilitating agents, Otolaryngol Head Neck Surg 122 (2000), 521-528.

[9] A.H. Clarke, U. Schonfeld and K. Helling, Unilateral examination of utricle and saccule function, J Vestib Res 13 (2003), 215-225.

[10] P.D. Cremer, G.M. Halmagyi, S.T. Aw, I.S. Curthoys, L.A. McGarvie, M.J. Todd, R.A. Black and I.P. Hannigan, Semicircular canal plane head impulses detect absent function of individual semicircular canals, Brain 121 ( Pt 4) (1998), 699-716.

[11] I.S. Curthoys, M.J. Dai and G.M. Halmagyi, Human ocular torsional position before and after unilateral vestibular neurectomy, Exp Brain Res 85 (1991), 218-225.

[12] L.E. Davis, Viruses and vestibular neuritis: review of human and animal studies, Acta Otolaryngol Suppl 503 (1993), 70-73.

[13] S. Esaki, F. Goshima, H. Kimura, S. Ikeda, S. Katsumi, K. Kabaya, N. Watanabe, M. Hashiba, Y. Nishiyama and S. Murakami, Auditory and vestibular defects induced by experimental labyrinthitis following herpes simplex virus in mice, Acta Otolaryngol 131 (2011), 684-691.

[14] M. Fetter and J. Dichgans, Vestibular neuritis spares the inferior division of the vestibular nerve, Brain 119 ( Pt 3) (1996), 755-763.

[15] J.M. Fishman, Corticosteroids effective in idiopathic facial nerve palsy (Bell's palsy) but not necessarily in idiopathic acute vestibular dysfunction (vestibular neuritis), Laryngoscope 121 (2011), 2494-2495.

[16] J.M. Fishman, C. Burgess and A. Waddell, Corticosteroids for the treatment of idiopathic acute vestibular dysfunction (vestibular neuritis), Cochrane Database Syst Rev (2011), CD008607.

[17] Y. Furuta, T. Takasu, S. Fukuda, Y. Inuyama, K.C. Sato and K. Nagashima, Latent herpes simplex virus type 1 in human vestibular ganglia, Acta Otolaryngol Suppl 503 (1993), 8589. 
[18] J.K. Goudakos, K.D. Markou, V. Franco-Vidal, V. Vital, M. Tsaligopoulos and V. Darrouzet, Corticosteroids in the treatment of vestibular neuritis: a systematic review and metaanalysis, Otol Neurotol 31 (2010), 183-189.

[19] J.K. Goudakos, K.D. Markou, G. Psillas, V. Vital and M. Tsaligopoulos, Corticosteroids and vestibular exercises in vestibular neuritis. Single-blind randomized clinical trial, JAMA Otolaryngol Head Neck Surg 140 (2014), 434-440.

[20] G.M. Halmagyi, Diagnosis and management of vertigo, Clin Med 5 (2005), 159-165.

[21] G.M. Halmagyi, S.T. Aw, M. Karlberg, I.S. Curthoys and M.J. Todd, Inferior vestibular neuritis, Ann N Y Acad Sci 956 (2002), 306-313.

[22] G.M. Halmagyi, K.P. Weber and I.S. Curthoys, Vestibular function after acute vestibular neuritis, Restor Neurol Neurosci 28 (2010), 37-46.

[23] K. Hasuike, T. Sekitani and Y. Imate, Enhanced MRI in patients with vestibular neuronitis, Acta Otolaryngol Suppl 519 (1995), 272-274.

[24] S.H. Jeong, H.J. Kim and J.S. Kim, Vestibular neuritis, Semin Neurol 33 (2013), 185194.

[25] M. Karlberg, M. Annertz and M. Magnusson, Acute vestibular neuritis visualized by 3-T magnetic resonance imaging with high-dose gadolinium, Arch Otolaryngol Head Neck Surg 130 (2004), 229-232.

[26] J.S. Kim and H.J. Kim, Inferior vestibular neuritis, J Neurol 259 (2012), 1553-1560.

[27] H.G. Macdougall, L.A. McGarvie, G.M. Halmagyi, I.S. Curthoys and K.P. Weber, The video Head Impulse Test (vHIT) detects vertical semicircular canal dysfunction, PLoS One 8 (2013), e61488.

[28] H.G. MacDougall, K.P. Weber, L.A. McGarvie, G.M. Halmagyi and I.S. Curthoys, The video head impulse test: diagnostic accuracy in peripheral vestibulopathy, Neurology 73 (2009), 1134-1141.

[29] G. Magliulo, G. Iannella, S. Gagliardi and M. Re, A 1-year follow-up study with CVEMPs, O-VEMPs and video head impulse testing in vestibular neuritis, Eur Arch Otorhinolaryngol (2014).

[30] P. Monstad, S. Økstad and A. Mygland, Inferior vestibular neuritis: 3 cases with clinical features of acute vestibular neuritis, normal calorics but indications of saccular failure, BMC Neurol 6 (2006), 45.

[31] H.P. Niedermeyer, G. Zahneisen, P. Luppa, R. Busch and W. Arnold, Cortisol levels in the human perilymph after intravenous administration of prednisolone, Audiol Neurootol 8 (2003), 316-321.

[32] O. Ozdoğmuş, O. Sezen, U. Kubilay, E. Saka, U. Duman, T. San and S. Cavdar, Connections between the facial, vestibular and cochlear nerve bundles within the internal auditory canal, J Anat 205 (2004), 65-75.

[33] L. Pollak, M. Book, Z. Smetana, M. Alkin, Z. Soupayev and E. Mendelson, Herpes simplex virus type 1 in saliva of patients with vestibular neuronitis: a preliminary study, Neurologist 17 (2011), 330-332.

[34] U. Reker, [Vestibular neuronitis and its differential diagnosis] HNO 29 (1991), 349-356.

[35] S.M. Rosengren, M.S. Welgampola and J.G. Colebatch, Vestibular evoked myogenic potentials: past, present and future, Clin Neurophysiol 121 (2010), 636-651.

[36] B.S. Shin, S.Y. Oh, J.S. Kim, T.W. Kim, M.W. Seo, H. Lee and Y.A. Park, Cervical and ocular vestibular-evoked myogenic potentials in acute vestibular neuritis, Clin Neurophysiol 123 (2012), 369-375. 
[37] P. Silvoniemi, Vestibular neuronitis. An otoneurological evaluation, Acta Otolaryngol Suppl 453 (1988), 1-72.

[38] S.A. Spear and S.R. Schwartz, Intratympanic steroids for sudden sensorineural hearing loss: a systematic review, Otolaryngol Head Neck Surg 145 (2011), 534-543.

[39] M. Strupp and T. Brandt, Vestibular neuritis, Semin Neurol 29 (2009), 509-519.

[40] M. Strupp, L. Jager, U. Muller-Lisse, V. Arbusow, M. Reiser and T. Brandt, High resolution Gd-DTPA MR imaging of the inner ear in 60 patients with idiopathic vestibular neuritis: no evidence for contrast enhancement of the labyrinth or vestibular nerve, J Vestib Res 8 (1998), 427-433.

[41] D. Theil, V. Arbusow, T. Derfuss, M. Strupp, M. Pfeiffer, A. Mascolo and T. Brandt, Prevalence of HSV-1 LAT in human trigeminal, geniculate, and vestibular ganglia and its implication for cranial nerve syndromes, Brain Pathol 11 (2001), 408-413.

[42] D. Vibert, R. Häusler and A.B. Safran, Subjective visual vertical in peripheral unilateral vestibular diseases, J Vestib Res 9 (1999), 145-152.

[43] I. Wegner, P.P. van Benthem, M.C. Aarts, T.D. Bruintjes, W. Grolman and G.J. van der Heijden, Insufficient evidence for the effect of corticosteroid treatment on recovery of vestibular neuritis, Otolaryngol Head Neck Surg 147 (2012), 826-831.

[44] M.S. Welgampola and J.G. Colebatch, Vestibulocollic reflexes: normal values and the effect of age, Clin Neurophysiol 112 (2001), 1971-1979.

[45] D. Zhang, Z. Fan, Y. Han, G. Yu and H. Wang, Inferior vestibular neuritis: a novel subtype of vestibular neuritis, J Laryngol Otol 124 (2010), 477-481. 